\title{
Concomitant Diagnosis of Primary Bone Marrow B-Cell Non-Hodgkin Lymphoma and Essential Thrombocythemia: A Case Report
}

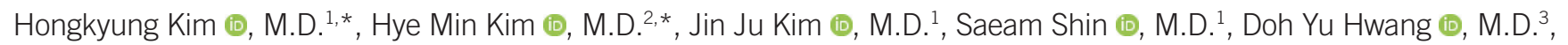 \\ Seung-Tae Lee $\mathbb{1}$, M.D. ${ }^{1}$, and Jong Rak Choi $\mathbb{0}$, M.D. ${ }^{1}$ \\ ${ }^{1}$ Department of Laboratory Medicine, Yonsei University College of Medicine, Severance Hospital, Seoul, Korea; ${ }^{2}$ Department of Pathology, Yonsei University \\ College of Medicine, Yongin Severance Hospital, Yongin, Korea; ${ }^{3}$ Division of Hemato-Oncology, Department of Internal Medicine, Yonsei University College of \\ Medicine, Yongin Severance Hospital, Yongin, Korea
}

\section{Dear Editor,}

Primary bone marrow lymphoma (PBML) is a rare form of lymphoma that originally arises in the bone marrow. The criteria for diagnosis of PBML include (a) pathological evidence of isolated bone marrow (BM) infiltration regardless of peripheral blood involvement, (b) absence of nodal or extranodal involvement, (c) no evidence of tumor formation, (d) no evidence of bony trabecular destruction on BM biopsy, and (e) exclusion of leukemia/lymphoma that involves the BM such as chronic lymphocytic leukemia (CLL)/small lymphocytic leukemia. The majority of PBMLs are confirmed to be diffuse large B-cell lymphomas (DLBCLs), with rare cases of other types of B-cell non-Hodgkin lymphomas (B-NHLs) reported as PBML [1]. The co-existence of PBML and other hematopoietic disorders has not been reported to date. We report a patient diagnosed as having PBML B-NHL and essential thrombocythemia (ET) concomitantly. The Institutional Review Board of Yongin Severance Hospital, Yon- gin, Korea, approved this study (IRB No. 9-2021-0005) and waived the need for informed consent.

A 72-year-old female with no specific medical history visited an outpatient clinic at Yongin Severance Hospital in August 2020 with a five-year history of intermittent palpitation. She exhibited leukocytosis and thrombocytosis with a white blood cell (WBC) count of $22.87 \times 10^{9} / \mathrm{L}$ ( $42 \%$ neutrophils, $51 \%$ lymphocytes, $5 \%$ monocytes, $2 \%$ eosinophils), hemoglobin of $132 \mathrm{~g} / \mathrm{L}$, and platelet count of $1,260 \times 10^{9} / \mathrm{L}$ in the complete blood count (CBC). Approximately $17 \%$ atypical lymphocytes, which had abundant cytoplasm and occasionally showed a cleaved nucleus, were observed on a peripheral blood smear (Fig. 1A, B). Peripheral blood flow cytometry (Beckman Coulter, Miami, FL, USA) revealed a monoclonal B-cell population with kappa light chain restriction. These cells were strongly positive for CD19, CD20, CD79b, and surface immunoglobulin (slg) kappa light chain, and negative for CD5, CD10, FMC7, CD23, CD38, CD138, and
Received: March 28, 2021

Revision received: July 19, 2021

Accepted: September 14, 2021

\section{Corresponding author: Saeam Shin, M.D.}

Department of Laboratory Medicine, Yonsei University College of Medicine, Severance Hospital, 50-1 Yonsei-ro, Seodaemun-gu, Seoul 03722, Korea Tel: +82-2-2228-2453, Fax: +82-2-364-1583, E-mail: saeam0304@yuhs.ac

Co-corresponding author: Doh Yu Hwang, M.D.

Division of Hemato-Oncology, Department of Internal Medicine, Yonsei

University College of Medicine, Yongin Severance Hospital, 363

Dongbaekjukjeon-daero, Giheung-gu, Yongin 16995, Korea

Tel: +82-31-5189-8965, Fax: +82-31-5189-8567, E-mail: hemayu78@yuhs.ac

*These authors contributed equally to this study.

\section{(i) $\$$}

\section{(C) Korean Society for Laboratory Medicine}

This is an Open Access article distributed under the terms of the Creative Commons Attribution Non-Commercial License (https://creativecommons.org/licenses/by-nc/4.0) which permits unrestricted non-commercial use, distribution, and reproduction in any medium, provided the original work is properly cited. 

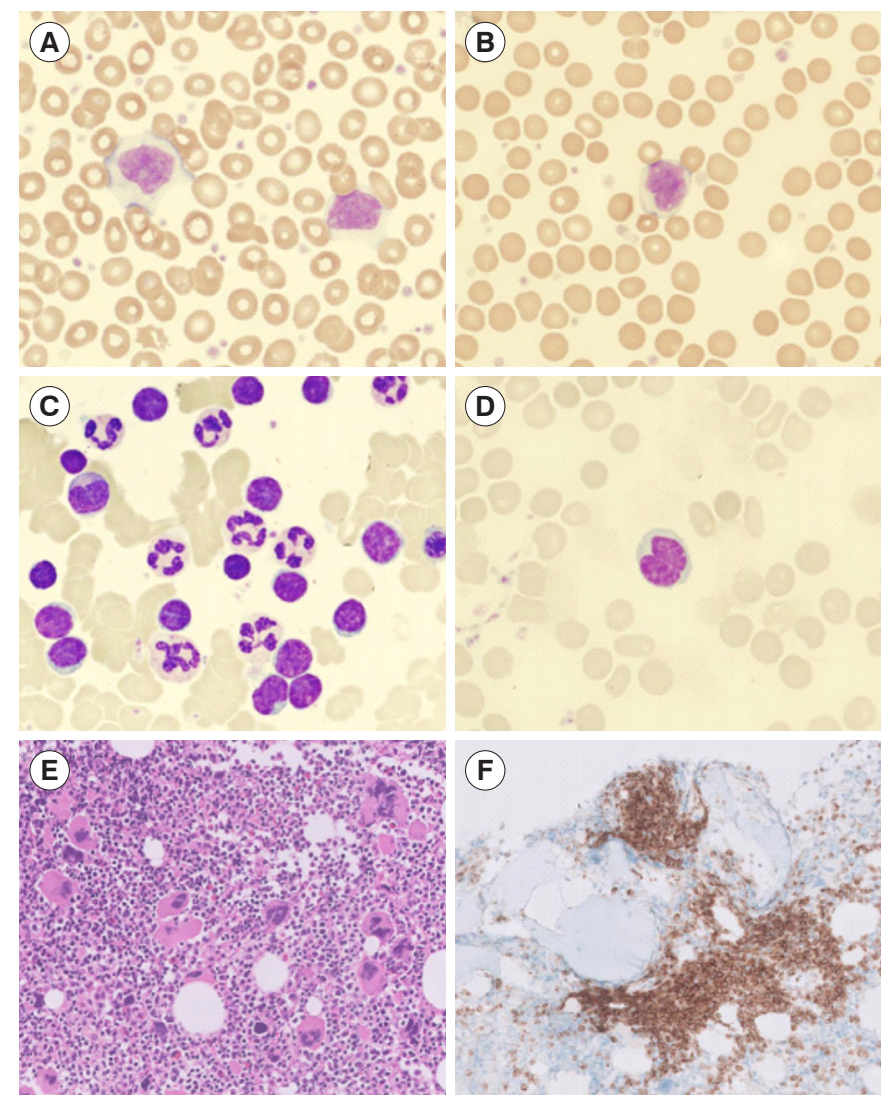

Fig. 1. Peripheral blood and bone marrow findings of primary bone marrow B-cell non-Hodgkin lymphoma with essential thrombocythemia. (A) Atypical lymphocytes with abundant cytoplasm and dense chromatin, and thrombocytosis on the peripheral blood smear (WrightGiemsa stain, $\times 100)$. (B) Atypical lymphocyte with a cleaved nucleus on the peripheral blood smear (Wright-Giemsa stain, $\times 100$ ). (C) Medium-sized atypical lymphocytes with an occasional indented or a cleaved nucleus on the bone marrow aspiration smear (WrightGiemsa stain, $\times 100$ ). (D) Atypical lymphocyte with a cleaved nucleus on the bone marrow aspiration smear (Wright-Giemsa stain, $\times 100$ ). (E) Markedly increased number of mature megakaryocytes with hyperlobulated nuclei often forming clusters (hematoxylin and eosin, $\times 20$ ). (F) Paratrabecular infiltrate of B-cell non-Hodgkin lymphoma in the bone marrow biopsy section (BCL2 stain, $\times 20$ ).

slg lambda light chain. This monoclonal B-cell population accounted for $62 \%$ of the total lymphocytes. Although BM aspiration was dry-tapped, some atypical lymphocytes, which were larger than the surrounding small lymphocytes and occasionally had an indented or cleaved nucleus, were observed on the BM aspirate smear (Fig. 1C, D). BM biopsy showed marked megakaryocytic hyperplasia, in which abnormal large and giant megakaryocytes with hypersegmented nuclei were occasionally observed (Fig. 1E). Multifocal non-paratrabecular and paratrabecular infiltrates of small to medium-sized lymphocytes were observed, which were immunohistochemically positive for BCL2 and CD20, and negative for BCL6, cyclin D1, ZAP-70, and CD138 (Fig. 1F). The atypical lymphocytes were focally positive for CD5 and CD23.

Clonality analysis, next-generation sequencing (NGS), karyotype analysis, and FISH were performed on the available BM aspirate. Clonal rearrangements in Ig heavy chain variable 3 family (IGHV3-23; $64.8 \%$ of total reads) and Ig kappa light chain variable 3 family (IGKV3D-20; 48.1\% of total reads) were demonstrated using LymphoTrack (Invivoscribe Technologies, San Diego, CA, USA). NGS was performed using the NextSeq 550Dx platform (Illumina, San Diego, CA, USA) and a custom panel targeting 497 genes associated with hematopoietic disorders; somatic variants of MYD88 p.Val217Phe (17.5\% variant allele frequency [VAF]) and JAK2 p.Val617Phe (16.3\% VAF) were detected (Fig. 2A, B). Trisomy 12 was suspected from copy number analysis using NGS data (Fig. 2C) and confirmed using Gbanding: 47,XX,+12[4]/46,XX [16]. The t(14;18)(q32;q21) was not detected by FISH. No paraprotein was detected in protein electrophoresis. There was no definite evidence of lymph node enlargement or splenomegaly in computed tomography scans of the neck, chest, abdomen, and pelvis. No discernible fluorodeoxyglucose uptake suggesting malignant processes was observed in positron emission tomography-computed tomography.

Given these ambiguous findings, several B-NHLs needed to be discriminated. There was more support for the possibility of atypical CLL or non-CLL lymphoma than for classical CLL based on the negative staining for CD5 and CD23 and detection of trisomy 12 . Morphologically aberrant lymphocytes and prolymphocytic cells are more frequent in atypical CLL with trisomy 12 [2]. However, CD5- and CD23-negative atypical CLL are very rare, and trisomy 12 is found in other lymphomas, such as mantle cell lymphoma and late-stage follicular lymphoma (FL) [2-4]. Among the several reported cases of a concomitant diagnosis of CLL and myeloproliferative neoplasm (MPN), none was a definite atypical CLL [5]. The possibility of high-grade FL was assessed since $C D 10$ and BCL 6 tend to diminish as the FL grade increases [6]. Similarly, $t(14 ; 18)(q 32 ; q 21)$ is infrequent in grade-3 FL [7]. Leukemic manifestation in PBML with the FL histological subtype is common [1]. More than $90 \%$ of lymphoplasmacytic lymphomas have MYD88 variants, most of which are p.Leu265Pro; however, p.Val217Phe was detected in this case. Owing to its low prevalence, the biological role of the MYD88 p.Val217Phe variant has not been clarified. However, it may contribute to lymphomagenesis related to nuclear factor kappa B activation, considering its recurrent nature and location within the MyD88 Toll/ interleukin-1 receptor domain [8]. DLBCL was morphologically 


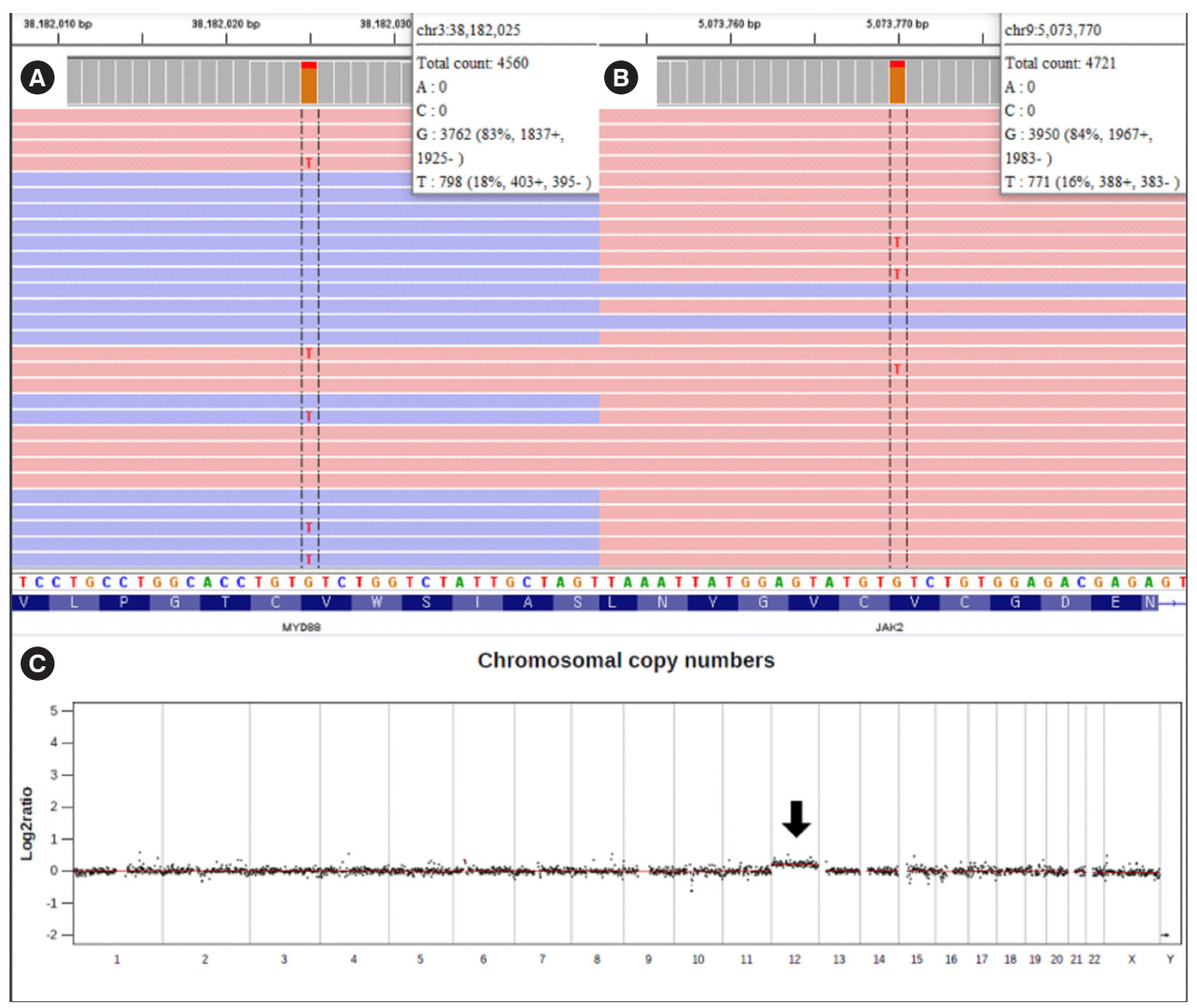

Fig. 2. Identification of somatic variants from bone marrow aspirates using the Integrative Genomics Viewer (Broad Institute, Cambridge, MA, USA). A custom targeted panel sequenced on the NextSeq 550Dx platform (Illumina, San Diego, CA, USA) detected (A) MYD88 p.Val217Phe (NM_002468.4:c.649G > T) at a variant allele frequency (VAF) of 17.5\% and (B) JAK2 p.Val617Phe (NM_004972.3:c.1849G > T) at a VAF of $16.3 \%$. (C) Trisomy 12 identified from off-target read analysis using next-generation sequencing (arrow). Data normalization for copy number variation was performed using germline data from skin fibroblast-derived DNA.

ruled out [3].

A watch-and-wait strategy was applied as an FL-oriented treatment, and oral hydroxyurea and aspirin were administered for ET. Ten months after the diagnosis, the patient did not show any symptoms, and the CBC indicated a WBC count of $12.43 \times 10^{9} / \mathrm{L}$ (79\% lymphocytes), hemoglobin of $98 \mathrm{~g} / \mathrm{L}$, and platelet count of $308 \times 10^{9} / \mathrm{L}$. Although a poor prognosis is generally predicted in PBML patients, the prognosis is significantly dependent on the histological subtype, which can explain the clinical outcome of our patient [1].

Patients with MPN have an increased risk of lymphomas. Although the relationship is uncertain, there may be shared pathogenic mechanisms occurring in early events of the hematopoietic process that increase the risk of both MPN and lymphoma [9]. Progressively increased genomic instability in MPN can lead to other lymphoma-related genetic variants [10].
In conclusion, we report the first case of a concomitant diagnosis of PBML and MPN. Although the type of B-NHL was never specified and a diagnosis of PBML cannot be confirmed if the $\mathrm{B}-\mathrm{NHL}$ is a lymphoma involving the BM (such as atypical CLL), this case shows that a comprehensive evaluation including molecular diagnostic tools can play a more important role in the diagnosis of lymphoma with unusual features and co-existing hematopoietic disorders.

\section{ACKNOWLEDGEMENTS}

None.

\section{AUTHOR CONTRIBUTIONS}

Kim H, Kim JJ, Lee ST, and Choi JR performed genetic and flow 
cytometric analyses. Kim HM performed the pathologic diagnosis. Kim H and Kim HM wrote the manuscript. Shin S and Hwang DY collected patient data, supervised the research, and reviewed and edited the manuscript.

\section{CONFLICTS OF INTEREST}

None declared.

\section{RESEARCH FUNDING}

This work was supported by the National Research Foundation of Korea (NRF-2019R1C1C1010916).

\section{ORCID}

Hongkyung Kim https://orcid.org/0000-0003-4185-1672

Hye Min Kim

Jin Ju Kim

Saeam Shin

Doh Yu Hwang

Seung-Tae Lee

Jong Rak Choi

https://orcid.org/0000-0002-2899-9480

https://orcid.org/0000-0001-9166-1848

https://orcid.org/0000-0003-1501-3923

https://orcid.org/0000-0002-0750-0011

https://orcid.org/0000-0003-1047-1415

https://orcid.org/0000-0002-0608-2989

\section{REFERENCES}

1. Martinez A, Ponzoni M, Agostinelli C, Hebeda KM, Matutes E, Peccatori
J, et al. Primary bone marrow lymphoma: an uncommon extranodal presentation of aggressive non-Hodgkin lymphomas. Am J Surg Pathol 2012;36:296-304.

2. Frater JL, McCarron KF, Hammel JP, Shapiro JL, Miller ML, Tubbs RR, et al. Typical and atypical chronic lymphocytic leukemia differ clinically and immunophenotypically. Am J Clin Pathol 2001;116:655-64.

3. Onaindia A, Medeiros LJ, Patel KP. Clinical utility of recently identified diagnostic, prognostic, and predictive molecular biomarkers in mature B-cell neoplasms. Mod Pathol 2017;30:1338-66.

4. Mohamed AN, Palutke M, Eisenberg L, Al-Katib A. Chromosomal analyses of 52 cases of follicular lymphoma with $t(14 ; 18)$, including blastic/ blastoid variant. Cancer Genet Cytogenet 2001;126:45-51.

5. Todisco G, Manshouri T, Verstovsek S, Masarova L, Pierce SA, Keating $\mathrm{MJ}$, et al. Chronic lymphocytic leukemia and myeloproliferative neoplasms concurrently diagnosed: clinical and biological characteristics. Leuk Lymphoma 2016;57:1054-9.

6. Horn H, Schmelter C, Leich E, Salaverria I, Katzenberger T, Ott MM, et al. Follicular lymphoma grade $3 \mathrm{~B}$ is a distinct neoplasm according to cytogenetic and immunohistochemical profiles. Haematologica 2011; 96:1327-34.

7. Godon A, Moreau A, Talmant P, Baranger-Papot L, Geneviève F, Milpied $\mathrm{N}$, et al. Is t(14;18)(q32;q21) a constant finding in follicular lymphoma? An interphase FISH study on 63 patients. Leukemia 2003;17:255-9.

8. Ngo VN, Young RM, Schmitz R, Jhavar S, Xiao W, Lim KH, et al. Oncogenically active MYD88 mutations in human lymphoma. Nature 2011; 3470:115-9.

9. Holst JM, Plesner TL, Pdersen MB, Frederiksen H, Møller MB, Clausen MR, et al. Myeloproliferative and lymphoproliferative malignancies occurring in the same patient: a nationwide discovery cohort. Haematologica 2020;105:2432-9.

10. Vannucchi AM, Masala G, Antonioli E, Chiara Susini M, Guglielmelli P, Pieri L, et al. Increased risk of lymphoid neoplasms in patients with Philadelphia chromosome-negative myeloproliferative neoplasms. Cancer Epidemiol Biomarkers Prev 2009;18:2068-73. 